\title{
Quality improvement project: identifying the barriers to accurate assessment and documentation of fluid status monitoring
}

\author{
Authors: Elizabeth Thompson, ${ }^{\mathrm{A}}$ Mousomi Batacharyya, ${ }^{\mathrm{A}}$ Abigail Bowers, ${ }^{\mathrm{A}}$ Grace Walker ${ }^{\mathrm{A} *}$ and Carlo Prina ${ }^{\mathrm{A}}$
}

\author{
Aim \\ To identify the barriers to accurate assessment and \\ documentation of fluid balance.
}

\section{Methods}

Fluid status monitoring was identified as an essential component in the care of patients with a presenting diagnosis of heart failure, sepsis, acute kidney injury and severe hyponatraemia. To measure the assessment and documentation of fluid status monitoring, data was collected from the electronic patient system (EPR) using a snapshot review on two wards over 48 hours pre- and post-intervention. Data collected included patient demographics, presenting diagnosis, early warning score, initial assessment of fluid status, if fluid monitoring was requested and fluid balance chart percentage completion.

Data was collected at baseline and following the plan, do, study, act (PDSA) cycle. PDSA cycle one included a session with 23 doctors and nurses that outlined the key aspects of fluid balance monitoring and provided room for discussion of barriers and mechanisms for improvement.

Questionnaires were used to assess nurses' and doctors' understanding of the importance of, barriers to and improvements in the assessment and documentation of fluid balance monitoring.

\section{Results}

The baseline data showed $30.5 \%$ of sample patients had no information documented on the fluid chart, $69.5 \%$ had some data documented and $0 \%$ were fully completed. The questionnaires reflected the data, showing $95 \%$ of nurses and $63 \%$ of doctors feel that fluid balance is managed suboptimally or poorly. The main barriers highlighted were time pressures, patient compliance, challenges with documentation on EPR, staff education and handover. The PDSA cycle one session highlighted the need for better communication and handover and staff education, and the issue of time constraints. Following PDSA cycle one there was no identified improvement in fluid balance documentation, with only $44 \%$ of patients having some fluid balance monitoring documented compared to $69.5 \%$.

\section{Conclusion}

Both quantitative and qualitative data indicate that fluid status monitoring is poorly documented. Barriers to accurate monitoring were identified as time constraints, poor handovers, poor staff education and confidence and patient compliance. This is a large topic which has the potential for significant improvement. Further PDSA cycles and interventions are required to improve documentation of fluid balance. Some planned future interventions are bulleted below.

$>$ Teaching sessions for doctors and nursing staff to improve confidence in assessing and documenting accurate fluid balance.

> A handover checklist to improve medical-nursing communication.

> Fortnightly snapshot data collection to generate a run chart to understand the random variation of fluid balance documentation and therefore clearly identify shifts and trends suggesting improvement.

\section{Conflicts of interest}

None declared. 